\title{
DTiGEMS+: drug-target interaction prediction using graph embedding, graph mining, and similarity-based techniques
}

\author{
Maha A. Thafar ${ }^{1,2}$, Rawan S. Olayan 1,3, Haitham Ashoor ${ }^{1,3}$, Somayah Albaradei ${ }^{1,4}$, Vladimir B. Bajic ${ }^{1}$, Xin Gao ${ }^{1}$, \\ Takashi Gojobori ${ }^{1,5}$ and Magbubah Essack ${ }^{1 *}$ (D)
}

\begin{abstract}
In silico prediction of drug-target interactions is a critical phase in the sustainable drug development process, especially when the research focus is to capitalize on the repositioning of existing drugs. However, developing such computational methods is not an easy task, but is much needed, as current methods that predict potential drugtarget interactions suffer from high false-positive rates. Here we introduce DTiGEMS+, a computational method

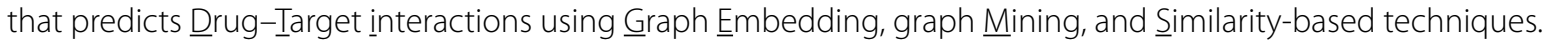
DTiGEMS + combines similarity-based as well as feature-based approaches, and models the identification of novel drug-target interactions as a link prediction problem in a heterogeneous network. DTiGEMS+ constructs the heterogeneous network by augmenting the known drug-target interactions graph with two other complementary graphs namely: drug-drug similarity, target-target similarity. DTiGEMS+ combines different computational techniques to provide the final drug target prediction, these techniques include graph embeddings, graph mining, and machine learning. DTiGEMS+ integrates multiple drug-drug similarities and target-target similarities into the final heterogeneous graph construction after applying a similarity selection procedure as well as a similarity fusion algorithm. Using four benchmark datasets, we show DTiGEMS + substantially improves prediction performance compared to other state-of-the-art in silico methods developed to predict of drug-target interactions by achieving the highest average AUPR across all datasets (0.92), which reduces the error rate by $33.3 \%$ relative to the second-best performing model in the state-of-the-art methods comparison.
\end{abstract}

Keywords: Drug repositioning, Drug-target interaction, Machine learning, Graph embedding, Heterogenous network, Similarity-based, Similarity integration, Bioinformatics, Cheminformatics

\section{Introduction}

The exorbitant costs, low success rates, and timeconsuming nature of the traditional experiment-based drug discovery processes have led to the incorporation of low cost in silico methods that can fast track

\footnotetext{
*Correspondence: magbubah.essack@kaust.edu.sa

${ }^{1}$ Computer, Electrical and Mathematical Sciences and Engineering Division (CEMSE), Computational Bioscience Research Center (CBRC), King Abdullah University of Science and Technology (KAUST), Thuwal, Kingdom of Saudi Arabia

Full list of author information is available at the end of the article
}

drug discovery and development [1]. In this regard, computational methods that predict drug-target interactions (DTIs) have been pursued to reduce the research focus area towards drugs that may be more viable. One of the initial steps in knowing which drugs to pursue is based on the drugs' ability to interact with a specific target protein to either enhance or inhibit its function [2]. However, there is a limited number of experimentally identified and validated DTI pairs. Thus, DTI prediction is an essential task in the early stage evaluation of potential novel drugs, and the search for

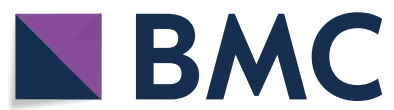

(c) The Author(s) 2020. This article is licensed under a Creative Commons Attribution 4.0 International License, which permits use, sharing, adaptation, distribution and reproduction in any medium or format, as long as you give appropriate credit to the original author(s) and the source, provide a link to the Creative Commons licence, and indicate if changes were made. The images or other third party material in this article are included in the article's Creative Commons licence, unless indicated otherwise in a credit line to the material. If material is not included in the article's Creative Commons licence and your intended use is not permitted by statutory regulation or exceeds the permitted use, you will need to obtain permission directly from the copyright holder. To view a copy of this licence, visit http://creativeco mmons.org/licenses/by/4.0/. The Creative Commons Public Domain Dedication waiver (http://creativecommons.org/publicdomain/ zero/1.0/) applies to the data made available in this article, unless otherwise stated in a credit line to the data. 
novel uses of existing drugs, i.e., drug repurposing [3]. To date, several approaches have been used to predict DTIs, but they all suffer from limitations and require substantially improved prediction performance.

One of the approaches used to predict DTIs, docking simulations [4, 5], requires the 3-dimensional (3D) structure of the protein target. However, such 3D structural information is not available for all targets, which limits the use of this approach. A second approach used to avoid this limitation when predicting DTIs is ligand-based [6, 7]. This approach predicts DTIs by comparing a candidate ligand with the known ligands of the proteins targeted. This approach suffers from low performance in cases where the targeted proteins have few known ligands. Subsequently, several computational methods have been developed to avoid the limitations of these traditional methods. That is, to a certain extent other computational methods may suffer from the same limitation but can incorporate features (such as different drug similarity, statistical and network features from DTIs heterogeneous graph, etc.) beyond ligand interaction features to improve prediction accuracy, and the methods can be designed for target-based drug discovery. Most of these methods use three types of information which are: drug-related information (e.g., chemical information for drugs), target-related information (e.g., protein sequences), or/and known DTI information. These methods can be grouped under three main categories namely: machine learning (ML)-based methods [8-12], deep learning (DL)-based methods [13-16] (DL is a branch of ML), and network-based methods [17-22]. Several comprehensive review articles summarized, analyzed, and compared the methods belonging to these categories [23-29].

ML-based methods were developed using a featurebased approach wherein feature vectors represent DTIs [26] and a similarity-based approach that uses the "guiltby-association" principle [30]. Some of the first works that successfully predicted DTIs based on supervised ML had been done by Yamanishi and coauthors using pharmacological, chemical, and genomic data [31-33]. Several methods developed based on these assumptions are summarized in [23], and most of these methods achieved promising results. Network-based methods formulate the prediction of DTIs as link prediction problem in a heterogeneous graph [19-21, 34-38]. For example, DASPfind [19] constructs a DTIs graph using a drug-drug similarity matrix, target-target similarity matrix, and known DTIs. After that, DASPfind ranks the DTIs based on their simple path scores to find the top $1 \%$ of DTIs. This method outperforms several networkbased methods when the single top-ranked predictions are considered using the benchmark DTI datasets,
Yamanishi_08 [33]. Since all of the drug-drug similarity (or target-target similarity), as well as DTIs, can be represented as adjacency matrices, matrix factorization approaches have recently been integrated with ML-based methods or/and network-based methods for prediction of DTIs [29, 39-43]. Graph embedding techniques [44, 45] applied on knowledge-graphs also improves the DTI prediction performance $[46,47]$ through the learning of low-dimensional feature representation of drugs or targets to be used in ML or DL based method. For example, DTINet [20] used matrix factorization as well as graph embedding approaches, to predict a novel DTIs from a heterogeneous graph. That is, DTINet combines several types of drug- and target protein-associated information, including drug-disease association, drugside effect associations, drug-drug similarity, drug-drug interactions, protein-protein interaction, protein-disease association, and protein-protein similarities to construct a full heterogeneous graph. DTINet constructs the objective function using matrix factorization and then learns a low-dimensional feature representation that captures the topological properties of each node in this heterogeneous graph. DTINet uses this feature representation to predict the DTIs. This method outperforms other state-of-the-art methods using the HPRD and DrugBank datasets. However, DTINet cannot predict the interaction of new drugs or targets, which is considered a limitation of this method [20].

Also, scaling these network-based methods to graphs with a massive number of nodes is not possible. Thus, recent use of DL techniques that are capable of dealing with graphs with a vast number of nodes, as well as large datasets and a large number of features has emerged for prediction of DTIs. These methods use DL techniques in the feature learning step or the prediction step $[13,14,48-$ 50]. DL-based methods work better with drug and target information from multiple sources for better performance since the information from a single source does not provide sufficient data for DL. For example, NeoDTI (NEural integration of neighbOr information for DTI prediction) [50], a DL-based method, integrates diverse information from 8 different sources (such as drug chemical structure similarity, drug side effects, and protein sequence similarity), to construct a heterogeneous network. NeoDTI learns feature representation for each drug and target by preserving the topological representations. NeoDTI is a powerful and robust tool compared to other recent DTIs prediction methods [50]. Other type of DL-based methods uses raw representations of input data such as SMILES or fingerprints of drugs and amino acid, or nucleotide sequences for proteins to develop an end-to-end learning model to predict DTIs [16, 51-53]. For example, DeepConv-DTI [51] applies a convolutional neural network $(\mathrm{CNN})$ to the 
amino-acid sequences of proteins and Morgan/Circular fingerprints that is a descriptor of the substructure of a drug after analyzing the molecule as a graph [54]. The $\mathrm{CNN}$ captures the local patterns for proteins that enrich their features. After that, the model concatenates the protein and drug features and feeds them to a deep, fully connected layer for the prediction of DTIs.

Here, to further improve prediction performance for DTIs, we propose a computational method that utilizes topological information as well as multiple drug similarities and target similarities. This method called DTiGEMS + (Drug-target interaction predic-

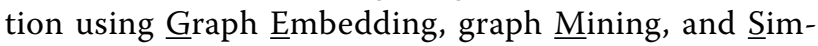
ilarity-based techniques) approaches DTI prediction as a link prediction problem in a heterogeneous graph. DTiGEMS+avoided limitations associated with the previously developed methods by integrating different techniques from graph embedding, graph mining, and fusing multiple similarities that reflect different information sources. DTiGEMS+ outperforms several stateof-the-art-methods using benchmark datasets in terms of AUPR performance metric. Our method proves its efficiency in the performance evaluation metrics and in

Table 1 Benchmark Yamanishi_08 datasets statistics

\begin{tabular}{lllll}
\hline Statistics & NR & GPCR & IC & Enzyme \\
\hline No. of drugs & 54 & 223 & 210 & 445 \\
No. of targets & 26 & 95 & 204 & 664 \\
Known DTIs & 90 & 635 & 1476 & 2926 \\
Unknown DTIs & 1314 & 20,550 & 41,364 & 292,554 \\
Sparsity ratio & 0.068 & 0.031 & 0.036 & 0.010 \\
\hline
\end{tabular}

predicting novel DTIs that are validated using literature and different databases.

\section{Materials \\ Benchmark datasets}

There are four gold standard datasets (Yamanishi_08) collected and compiled by [33], which were commonly used as benchmark datasets to evaluate the performance of recently developed DTIs prediction methods. Each of the four datasets, namely Enzyme (E), Ion channel (IC), G-protein-coupled receptor (GPCR), and Nuclear receptor (NR), represents one of the significant families of protein targets. These benchmark datasets are publicly available at http://web.kuicr .kyoto-u.ac.jp/supp/yoshi/drugtarge. Table 1 provides the statistics for all datasets used in this study. The sparsity ratio represents the number of known DTIs divided by the number of unknown DTIs and reflects the imbalanced nature between positive and negative samples (see Table 1).

\section{Data preprocessing and similarity calculations}

Starting from the "guilt-by-association" principle that similar drugs may share similar targets and vice versa as illustrated in Fig. 1, we incorporate and utilize several information sources in our approach in the form of different similarity measures (i.e., kernels) between each drug pair or target (i.e., protein) pair. Several drug-drug similarity and target-target similarity are calculated to capture different sources information from different points of view.

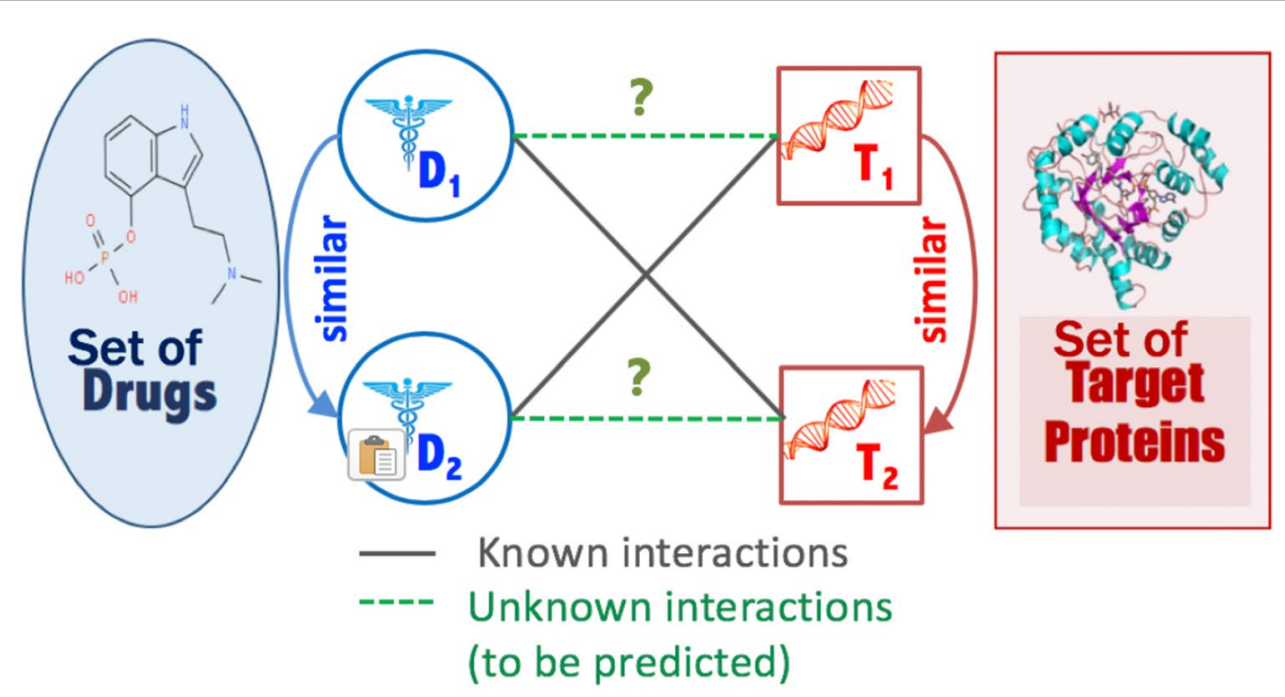

Fig. 1 DTIs prediction problem depiction 


\section{Multiple drug-drug similarities}

Following the [10] study, we computed or retrieved 10 representations or characteristics that can be used to determine drug similarity. That is, six different representations were used for the similarity between drugs based on the chemical structure (SDF, MOL, or SMILES formats) including the SIMCOMP similarity (provided by [33]), and the Spectrum [55], Marginalized [56], Lambda-k [55], Tanimoto, and Min-Max-Tanimoto [57] similarity matrices, calculated using Rchemcpp [58], KEGGREST [59], and ChemmineR [60]. Similarly, three different representations, retrieved from the [10] study, were used for the similarity between drugs based on the side effects, including SIDER [61], AERS-freq [62], and AERS-bit [62] similarity matrices. The tenth drug similarity was calculated based on the gaussian interaction profile (GIP), introduced in [63], that projects the drug-target network structure in the form of a network interaction profile. Additional file 1: Table S1 summarizes all the drug similarity matrices with their names and sources.

\section{Multiple target-target similarities}

Similar to drug similarities, we computed or retrieved 10 target similarity matrices from the [10] study. Seven different representations mirror the similarity between targets based on the amino-acid protein sequence including the normalized Smith-Waterman (SW) scores [64], and two Spectrum similarity matrices (with k-mers equal to 3 and 4), and four Mismatch similarity matrices (with different parameters of k-mers length and the number of maximal mismatch per $\mathrm{k}$-mer) recalculated using the R packages, KEGGREST [59], and KeBABS [65]. Gene Ontology (GO) similarity matrices based on the GO terms were calculated using the GO.db and annotate $\mathrm{R}$ packages [66]. Protein-protein interaction (PPI) similarity that mirrors the shortest distance between each target pair in the PPI network, obtained from [10] study. The GIP is calculated for the targets as we did for the drugs. Additional file 1: Table S1 summarizes all the target similarity matrices with their names and sources.

\section{Methods}

\section{Problem formulation}

In this work, we adopt a network-based approach. We define a weighted heterogeneous graph represented by the DTIs network augmented with the drug-drug similarity graph and target-target similarity graph. This defined graph $G(V, E)$ consists of a set of drugs $D=\left\{d_{1}\right.$, $\left.d_{2}, \ldots, d_{n}\right\}$ of $\mathrm{n}$ drug nodes, and set of targets $T=\left\{t_{1}, t_{2}, \ldots\right.$, $\left.t_{m}\right\}$ of $\mathrm{m}$ target nodes. DTI graph $\mathrm{G}$ contains three types of edges. The first type of edge represents the interaction between drug and target nodes, and edges from this type were assigned a weight of 1 . The second and third types of an edge represent the similarity between drugs and the similarity between targets, and these types of edges are assigned weights that have a real value between 0 and $1(0,1]$. Given graph $\mathrm{G}$, we define the DTI prediction problem as a link prediction problem, where the goal is to predict unknown true interactions (represented by links) between drugs and targets (see Fig. 1).

We constructed all possible pairs between drugs and targets by generating a "negative sample". Generating this "negative sample" involved creating connections (i.e., unknown interaction) between drug nodes and target nodes that have no edges. Thus, similar to other existing computational approaches, we used a reliable set of DTIs as positive interactions, and randomly generated drugtarget pairs to generate negative DTIs. That is, DTIs existing in the positive set were removed from the randomly generated drug-target pairs to generate negative DTIs. This is done, since there are not enough experimentallyvalidated negative DTIs available for most sets of drugs and targets. In our work, we believe that random pairing is probably more likely to be well-represented for negative DTIs since the ratios of known (positive) versus nonexisting (not known, negative) DTIs is very small. Then, we extracted features for each drug-target pair using different techniques. The feature vector is represented by $X=\left\{x_{1}\right.$, $\left.x_{2}, \ldots, x_{n^{* m}}\right\}$ and their labels $Y=\left\{y_{1}, y_{2}, \ldots, y_{n^{*} m}\right\}$ where $n^{*} m$ is equal to the number of drugs multiplied by the number of targets that represents the number of all possible (drug, target) pairs. If there is a known interaction for the drug-target pair, the class label $y$ for this pair is equal to $1(y=1)$; otherwise, the class label is equal to zero $(y=0)$. Thus, it is a binary classification task. The aim is to find novel DTIs with high accuracy and low false-positive rate. The proposed method integrates several techniques from the perspective of ML similarity-based, feature-based, and graph-based methods for DTI prediction.

\section{Similarity-based algorithms Similarity integration technique}

We used several integration functions to combine the multiple similarities matrices, including summing them up to take the average (AVG), taking the geometric mean (GeoM), choosing the maximum similarity value (MAX), or applying the similarity network fusion algorithm (SNF) that was introduced by [67] (see Fig. 2). Each similarity measure is represented by a square matrix, as shown in Fig. 2. The SNF first constructs a sample similarity network for each of the similarity matrices (i.e., drugs represent network nodes, and the similarity represents the networks' weighted edges but without self-loop edges, and the same thing is done for the target proteins separately). Then, SNF uses a nonlinear method that iteratively integrates these networks by updating each of the 


\section{3 similarity matrices}

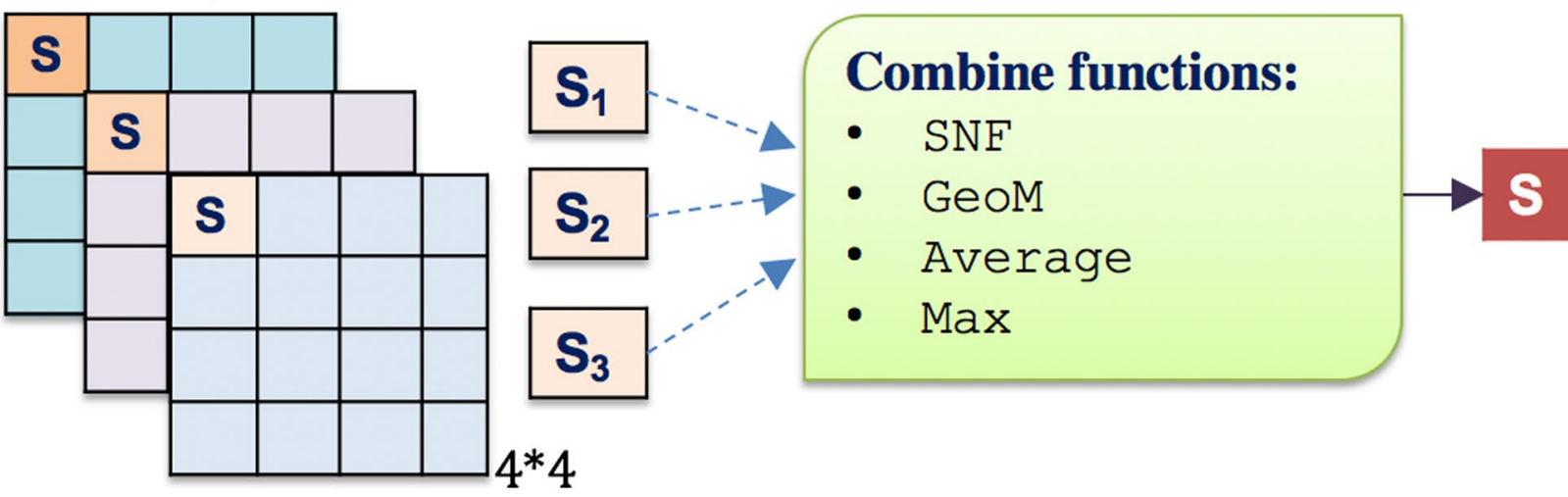

Fig. 2 Integrating multiple similarities using different functions

networks with the information from the other networks (making the similarity criteria more discriminant with each step) using K-nearest neighbor (KNN). SNF stops when networks converge to a single network after a few iterations. More details about the SNF function and its parameters are explained in [67].

\section{Similarity selection technique}

To select the optimal subset of similarities that are robust and should improve the prediction task, we applied a forward similarity selection (FSS) procedure as a heuristic process to obtain the best similarity combination. FSS follows the same concept as forward feature selection, where a pair of drug-drug similarity and target-target similarity are added in a "greedy fashion" until one observes no improvement in the performance. In more detail, the input for the FSS algorithm is a list of all drug-drug similarity matrices (all_DDsim) and a list of all target-target similarity matrices (all_TTsim). The algorithm initializes two other lists, one empty list (DDsim) to add selected drug-drug similarity matrix as well as another empty list (TTsim) to add selected target-target similarity matrix. FSS starts with a one drug-drug similarity and one targettarget similarity and do this iteratively for all possible combinations of the lists: all_DDsim and all_TTsim and then report the results of all these combinations. The pair of drug-drug similarity and target-target similarity with the best results are chosen to be the first similarity fixed in the DDsim and TTsim. In the second round, we have one fixed drug-drug similarity and target-target similarity, and we add another single similarity to both drug-drug and target-target lists and fuse them using SNF, and report all results. Again, the similarity with the best results is added and fixed in DDsim and TTsim. We repeat these steps, and each round, we add similarities with the best result to the selected similarity sets and fuse them and only stop the repetitions when the results converge (i.e., have no improvements). These "fused" results are used to generate graph $\mathbf{G 1}$.

\section{Graph embedding for feature learning}

Given a graph $G=\{V, E\}$, a graph embedding method will transform graph $\boldsymbol{G}$ into $R^{d}$ where $d \ll|v|$. In simple words, the graph embedding method will represent each node in the graph with a feature vector which is much smaller than the actual number of nodes in the graph while preserving the graph structure and properties [45]. To do this, we used the algorithmic framework of node2vec [68], to apply feature representational learning on the full heterogeneous graph $\mathrm{G}$ that consists of the training part of known DTIs after hiding the DT edges in the test data, drug-drug similarity matrix (DD sim), and target-target similarity (TT sim) (Fig. 4).

To reduce the node2vec processing time, we removed the weak edges that do not provide any informative meaning, from the drug-drug and target-target similarity graphs. That is, for each drug (or target), we kept the top-k similar drugs (or targets) and removed all other edges. After removing all weak edges, the drug-drug and target-target KNN similarity graphs are augmented with the training part of DTIs and fed into the node2vec model.

After applying node2vec on the heterogeneous graph $\mathrm{G}$ to learn feature representation for each drug and target, cosine similarity is calculated between each pair of drugs and each pair of targets to construct two new matrices. These matrices are, $M_{d}$, drug-drug similarity matrix of size $n * n$ where $n$ is the number of drugs, and $M_{t}$, target-target similarity matrix with size $m * m$, where $m$ is the number of 
targets; they are used to construct graph G2. After calculating cosine similarity, new edges could appear between pairs of drugs (or targets) based on the structural and topological similarities that don't have high similarity in the main graph with KNN drug similarity and KNN target similarity, which further prevents the missing of important information.

To utilize and obtain the optimal set of node2vec hyperparameters, grid search algorithm is applied on the validation data. The values of the hyperparameters that are tested on the training data are as follows: Return parameter $p$ (controls the likelihood of immediately revisiting a node in the walk) and In-out parameter $q$ (allows the search to differentiate between "inward" and "outward" nodes) can be one of the values $\{0.25,0.5,1,2,4\}$ as specified in node2 vec work, dimension $d$ can be $\{16,32,64,128\}$, number of walk per source, num-walk tried values $\{5,10,15,20\}$, and walk-length takes range based on the size of the graph. For example, in NR dataset we tested values of walk-length starts with 10 and add 5 each time until reach 60 \{10:5:60\}, while in Enzyme dataset which its graph much bigger we tested the values $\{50: 10: 160\}$. The walk parallelizes by assigning the hyperparameter workers to several workers based on the CPU core number. Additional file 1: Table S4 provides the optimized hyperparameter values for each dataset.

\section{Graph-based feature extractions for drug-target path scores}

At this stage, the two heterogeneous weighted graphs G1 and $\mathbf{G} 2$ are used to extract graph-based features. Multiple path scores between each drug-target pair for each graph is used to mirror these features (see Fig. 4). The path score is calculated for each simple path starting from the source node (i.e. drug) and ending with the target node (i.e. target protein) for each drug-target pair using path score, similar to the DASPfind path score introduced by [19] using the following formula:

$$
\operatorname{score}\left(d_{i}, t_{j}\right)=\sum_{p=1}^{n} \prod\left(P_{\text {weights }}\right)
$$

where $P=\left\{p_{1}, p_{2}, \ldots, p_{n}\right\}$ is the set of paths that connect drug $_{\mathrm{i}}$ to target ${ }_{\mathrm{j}}$. In our study, we reduce the computational costs by limiting the path length to be less than or equal to three (i.e., path length $=2$ or 3 ). Thus, there are six potential path structures $\mathrm{Ch}=\{C 1, C 2, C 3, C 4, C 5, C 6\}$ (referred to as path categories in [21,34]); each starting with a drug node, ending with a target node, and each node in the path appearing only once (no cycling). The six path structures include the two path structures with path length $=2(\mathrm{C} 1:(\mathrm{D}-\mathrm{D}-\mathrm{T})$ and $\mathrm{C} 2:(\mathrm{D}-\mathrm{T}-\mathrm{T}))$, and four path structures with length $=3(\mathrm{C} 3:(\mathrm{D}-\mathrm{D}-\mathrm{D}-\mathrm{T}), \mathrm{C} 4$ : (D-T-T-T), C5: (D-D-T-T), and C6: (D-T-D-T)). We calculated two features for each path structure by determining, 1/the Sum of all meta-path scores for each path structure, and 2/the Max score of all meta-path scores under each path structure. A meta-path is all paths that have the same path structure, and the meta-path score is the product of all the edge weights from the start drug node to the ending target node in the path structure. $R_{i j h}$ denotes the set of paths between a pair of drug $_{\mathrm{i}}$ and target $_{j}$. The equations used to determine the features for each path structure are defined and described in Table 2.

To ensure longer paths are not disadvantaged in our method, each (Max or Sum) path score is calculated independently, where each score considers all sets of paths that belong to a specific path structure. Thus, scores from different path structures are not mixed together in one feature. Also, scores are further normalized using min max normalization to make sure that features are equally treated by the classifier.

We extract 12 features for each (drug, target) pair and for each constructed heterogeneous graph (i.e., G1 and G2) (explained in detail in "DTIs predictive model" section) that are combined to form a 24-dimensional feature vector. Figure 3 provides an example that illustrates the graph-based feature extraction process through the D-D-D-T path structure.

To speed up the running time, we obtain the path scores by applying 3D matrix multiplication. We represented each graph with an adjacency matrix, that includes the drug-drug adjacency matrix (DD_sim), target-target adjacency matrix (TT_sim), and drug-target interaction matrix (DTI). The path score for each path structure is represented by matrix multiplication operation as introduced in [69]. The length of each path

Table 2 The equations used to determine path structure features

\begin{tabular}{ll}
\hline Score description & Equation \\
\hline $\begin{array}{l}\text { The meta-path score is the product of all the edge weight scores from the start drug node to the } \\
\text { ending target node in each path structure }\end{array}$ & $\operatorname{score}\left(d_{i}, t_{j}, h, q\right)=\prod_{\forall e_{x} \in P_{q}}\left(w_{x}\right)$ \\
The sum of all meta-path scores for each path structure (Sum feature) & SumScore $\left(d_{i}, t_{j}, h\right)=\sum_{\forall P_{q} \in R_{i j h}} \operatorname{score}\left(d_{i}, t_{j}, h, q\right)$ \\
The max path score is the highest meta-path score under each path structure (Max feature) & MaxScore $\left(d_{i}, t_{j}, h\right)=\operatorname{MAX} \forall P_{q} \in R_{i j h}\left(s c o r e\left(d_{i}, t_{j}, h, q\right)\right)$
\end{tabular}




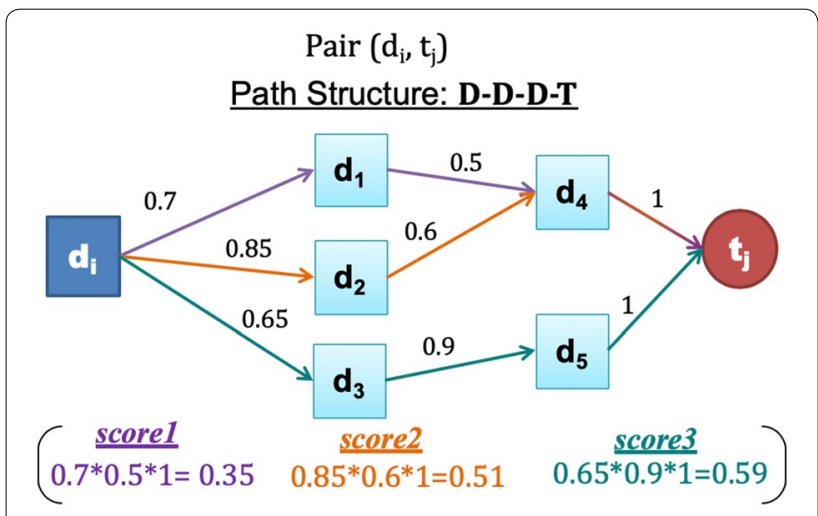

Feature 1: $\boldsymbol{\Sigma}$ (meta-path scores) $=1.45$

Feature 2: Max (meta-path scores) $=0.59$

Fig. 3 An illustration of Sum and Max scores for a D-D-D-T path structure

structure is equal to the number of multiplied adjacency matrices. Thus, if the path length $=3$, such as D-T-T$\mathrm{T}, 3$ matrices are multiplied to obtain the same results.
For Sum score features, regular matrix multiplication is enough to be performed, and the resulting matrix represents the sum features. However, for the Max scores feature, a 3D matrix multiplication is performed to obtain the multiplied value (i.e. the multiplied edge scores) for each path structure, and then choose the max score instead of summation process. Additional file 1: Table S3 provides the corresponding matrix multiplication to each path structure, as well as the semantic meaning for each path structure.

\section{DTIs predictive model Feature selection}

The accuracy of a predictive model relies on identifying the essential features of the examined dataset. Thus, empirical analysis and many experiments were performed (using a concept similar to the forward feature selection method), to identify a collection of the most relevant features for this classification task. Analyzing the performance involved removing one or a combination of features. Consequently, after applying the feature

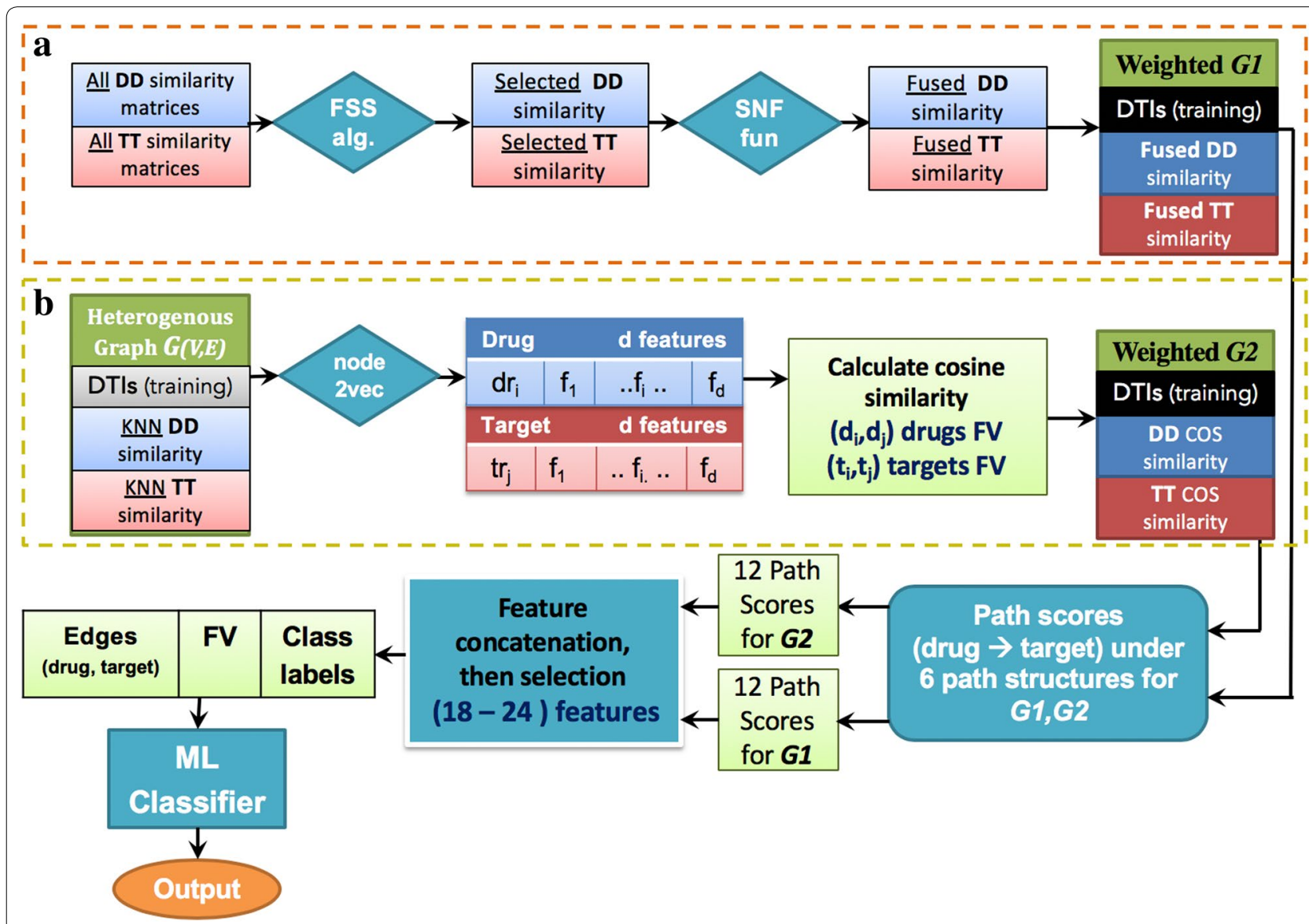

Fig. 4 DTiGEMS + prediction Framework. DTIs: drug-target interactions; DD: drug-drug; TT: target-target; FV: feature vector; FSS alg.: forward similarity selection algorithm; SNF fuc: similarity network fusion function; COS similarity: cosine similarity; ML: machine learning 
selection step, the dimension of the feature vectors fed into the predictive model reduced from 24 to range between 18 and 20 features based on the dataset.

\section{Sampling techniques for imbalanced data}

To deal with the number of unknown DTIs being much larger than the number of known DTIs, as shown in Table 1, we applied oversampling techniques on the training data to adjust the data to be balanced. That is, Random oversampling [70] or the Synthetic Minority Over-sampling Technique (SMOTE) [71] were applied to the minority class (i.e., positive known DTIs) to have the same number as the major class (negative unknown DTIs) in training data. The implementation of both techniques was done using the imbalanced-learn python package [72]. Random oversampling contributes to the best classification performance in some datasets, while SMOTE contributes better in other datasets.

\section{Classification model}

Supervised machine learning model is used to predict DTIs based on three different classifiers for each dataset mainly: Artificial neural network (NN) also called multilayer perceptron (MLP) [73], random forest (RF) [74], and adaptive boosting (Adaboost) [75] classifiers using scikit-learn implementation [76]. In our work, for each classifier used for a specific dataset, the most critical parameters are optimized using the training datasets to improve the classifier performance. Example of these parameters, for the $\mathrm{NN}$ classifier, include activation function, the size of hidden nodes and layers, and batch size, while the RF classifier parameters include, the number of trees, the maximum depth of the trees, the number of features to consider when looking for the best split, the function to measure the quality of a split, and others. On the other hand, we used Adaboost to boost the decision tree classifier, so that similar parameters similar to those used in the RF is optimized. The input to these classifiers is the feature vector $X$ of all possible drug-target pairs with their labels $Y$.

\section{The DTiGEMS + framework}

Figure 4 provides the stepwise framework used to obtain the feature vector, $X$, for all drug-target pairs that are used to predict the missing edges (unknown DTIs to be positive interaction). We generated $X$ from two graphs (G1 and G2). We generated graph G1 as follows: (1a) applied the FSS procedure to all DD and TT similarities, to select the optimal similarities subset, (2a) integrated these selected similarities using the SNF algorithm, then, (3a) used the DD fused similarity, TT fused similarity, and the DTI training part to construct the heterogeneous graph G1. Simultaneously, we prepared the second graph
G2 as follows: (1b) applied node2vec to the initial heterogeneous graph $\mathrm{G}$, to generate the feature representations for each node, $(\mathbf{2 b})$ calculated cosine similarity for each drug-drug pair and target-target pair, then, (3b) used the DD cosine similarity, TT cosine similarity, and the DTI training part to construct the heterogeneous graph G2. As a fourth step (4), for both graphs $\mathbf{G 1}$ and $\mathbf{G 2}$, we extracted 12 path scores for each graph, from six path structures. Then as a (5) and (6) step, feature selection was applied to eliminate weak features, followed by the generated feature vector, $X=\left\{x_{1}, x_{2}, \ldots, x_{n^{*} m}\right\}$, with their labels $Y=\left\{y_{1}, y_{2}, \ldots, y_{n^{*} m}\right\}$ for all drug-target pairs, being fed into the supervised ML prediction model using NN, RF, or Adaboost classifiers. Then the output of the classifier is the class label, which is either a positive or negative label.

\section{Evaluation methods \\ Evaluation metrics}

To evaluate the prediction accuracy, the area under the receiver operating characteristic (ROC) curve (AUC) [77], as well as the area under the precision-recall curve (AUPR) [77], are calculated on the testing data. To determine the AUC and AUPR, we calculated the false positive rate (FPR), recall (also called true positive rate (TPR) or sensitivity), and precision (also called positive predictive value) [78], based on true positive (TP), false positive (FP), true negative (TN) and false negative (FN) values, as shown in Eqs. 2, 3, and 4, respectively.

$$
\begin{aligned}
& F P R=\frac{F P}{T N+F P} \\
& \text { Recall }=T P R=\frac{T P}{T P+F N} \\
& \text { Precision }=\frac{T P}{T P+F P}
\end{aligned}
$$

The ROC curve is constructed using different recall, and FPR values of different thresholds, to calculate the AUC. AUPR is calculated based on different precision and recall values at different cut-offs that used to construct the curve, and then the area under this curve is calculated. The closer the value of AUC and AUPR are to 1 , the better the performance is. For highly imbalanced (i.e., number of unknown DTIs is much higher than the known DTIs) data, the AUC is considered an over-optimistic evaluation metric for prediction of DTIs, while AUPR is thought to provide better assessment in such imbalance data cases, because it separates the predicted scores of true interactions from the predicted scores of unknown interaction. Thus, we 
use AUPR as the significant evaluation metric and for the comparison with state-of-the-art methods, but also calculate the error rate (ER), and the relative error rate reduction for the best performing model compared to the second-best performing model $(\triangle E R)$, defined in Eqs. 5, and 6, respectively:

$$
\begin{aligned}
& E R=1-A U P R \\
& \Delta E R=\frac{\left(E R_{2}-E R_{1}\right)}{E R_{2}}
\end{aligned}
$$

\section{Experimental settings}

For DTiGEMS+ prediction performance evaluation, we performed tenfold cross-validation $(\mathrm{CV})$ on each benchmark dataset separately. The data was randomly partitioned into 10 subsets in a stratified fashion where each subset must include the same percentage of negative and positive samples (i.e., known and unknown DTIs). We kept aside 1 subset of the data for testing and used the remaining 9 subsets to train the model. This process was repeated 10 times to have each subset of the data to be in the test part and the other 9 subsets to train the model. This CV is called a random $\mathrm{CV}$ setting where random drug-target pairs are removed to be in test data. The AUPR and AUC calculated for each fold, then the average AUPR and the average AUC of the tenfolds are reported. Here, we removed the corresponding edges to all known DTIs that are in the test set from all constructed graphs in our framework, including G, G1, and G2.

\section{Results and discussion}

Here, we compare the DTI prediction performance between our method and the state-of-the-art methods and validate the newly predicted DTIs using several databases. We also highlighted several possible characteristics that could be boosting the prediction performance of the DTiGEMS + method compared to other methods.

\section{DTI prediction performance of DTiGEMS+}

To evaluate our method, we compare the DTI prediction performance of DTiGEMS+and seven state-of-the-art methods using the benchmark Yamanishi_08 datasets. The state-of-the-art methods include TriModel [46], DDR [21], DNLMF [43], NRLMF [39], KronRLS-MKL [10], RLS-WNN [79], and BLM-NII [80]. We chose these methods to give a broad perspective of DTiGEMS+ DTI prediction performance compared to network-based (i.e., graph-based) and or matrix factorization-based methods, as they are all ML similarity-based methods that use prior knowledge to integrate multiple similarity measures from different sources.

To provide a fair comparison of DTI prediction performances, we used the same benchmark datasets, tenfold $\mathrm{CV}$ random setting, evaluation metrics, and optimal parameters provided by each method. Our method DTiGEMS + outperforms all other methods by achieving the best performance across all benchmark datasets (highest averageAUPR $=0.92$, highest averageAUC $=0.99$ ), which is $4 \%$ higher averageAUPR and $1 \%$ higher averageAUC than the second-best method (TriModel) (see Table 3). It also has the best average ranking position across all datasets (the lower ranking position, the better is the method). In Table 3, the best results in each row are indicated in italic font with underline, while the second-best results are only in italic font.

For each dataset, DTiGEMS+(in blue) performs better in terms of AUPR 0.88(0.094), 0.86(0.031), $0.96(0.013)$, and $0.97(0.005)$ for the NR, GPCR, IC, and $\mathrm{E}$ datasets, respectively, and the values between brackets are the standard deviations of AUPR in tenfolds CV. DTiGEMS+ outperforming the second-best method (TriModel, in purple) by $4 \%, 6 \%, 3 \%$, and $2 \%$ for the NR, GPCR, IC, and E datasets, respectively (shown in Fig. 5). DTiGEMS+also outperformed the other methods in terms of AUC for each dataset except TriModel that have the same performance for the NR, IC, and E datasets (see Additional file 1: Table S5). Figure 5 further shows better DTI prediction performance was achieved using the

Table 3 Average scores for the AUPR, AUC, and ranking position for all comparison methods across all benchmark

\begin{tabular}{|c|c|c|c|c|c|c|c|c|}
\hline Methods & BLM-NII & KronRLS & RLS-WNN & NRLMF & DNILMF & DDR & TriModel & DTiGEMS+ \\
\hline Average AUPR & 0.68 & 0.73 & 0.76 & 0.80 & 0.78 & 0.87 & 0.88 & $\underline{0.92}$ \\
\hline Average AUC & 0.92 & 0.90 & 0.96 & 0.95 & 0.95 & 0.96 & 0.98 & $\underline{0.99}$ \\
\hline $\begin{array}{l}\text { Average of the ranking } \\
\text { position across all datasets }\end{array}$ & 8 & 7 & 6 & 4 & 5 & 3 & 2 & 1 \\
\hline
\end{tabular}
datasets

We rounded-off all results to two decimal places. The italic font with underline indicates the best result in each category, while the italic font only indicates the second-best result 


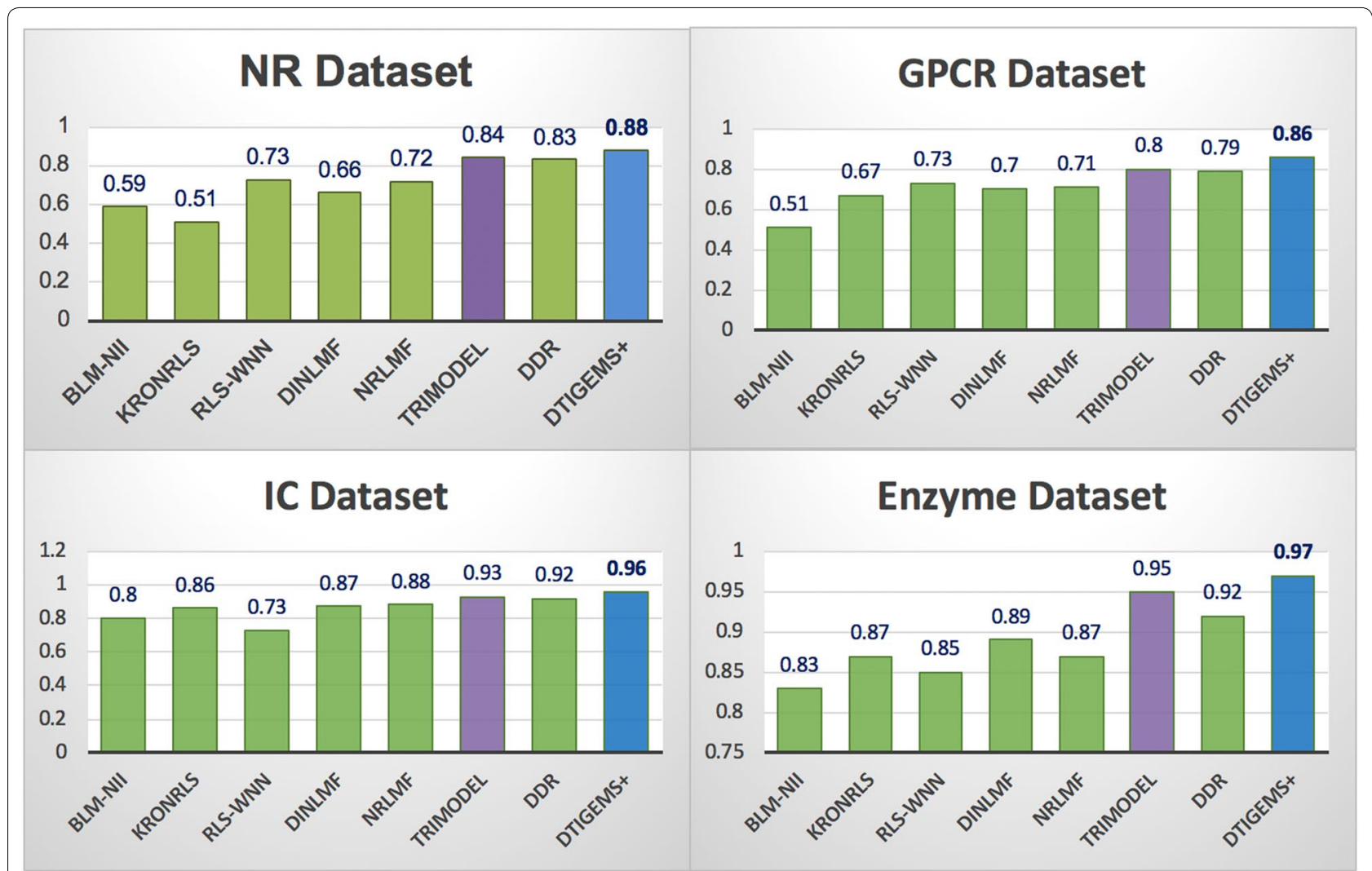

Fig. 5 Comparison results for DTiGEMS+ and other methods in terms of AUPR using the Yamanishi_08 datasets. The best performing method is indicated in blue, the second-best method in purple, and all other methods in green

IC and E datasets; this may be attributed to these datasets having a more extensive set of positive interaction data the models can use to refine the features used for prediction. Moreover, based on individual AUPR values reported from tenfold $\mathrm{CV}$ experiments, we calculated the statistical significance in terms of the performance improvement of our method relative to the next best method TriModel using Wilcoxon test which is a nonparametric statistical test that compares two paired groups (refers to the Rank sum test, or the Signed Rank test). As a result, we demonstrate that DTiGEMS+ shows significant statistical difference with probability values (P-values) $<0.05$ obtained over GPCR, IC, and E datasets as 0.04, 0.004 and 0.002, respectively, except for NR dataset which has $\mathrm{P}$-value $>0.05$.

Two other evaluation metrics are used to gain more insights about the prediction performance improvement of our method DTiGEMS + over the other methods which are: error rate (ER), and the relative error rate reduction for the best performing method compared to the second-best performing method $(\Delta \mathrm{ER})$, defined in Eqs. 5 and 6 , respectively. Table 4 provides a comparison of the ERs for DTiGEMS+, as the best performing method,

\begin{tabular}{|c|c|c|c|}
\hline Datasets & $\begin{array}{l}\text { ER }_{1} \\
\text { of DTiGEMS+ (\%) }\end{array}$ & $\begin{array}{l}\text { ER }_{2} \\
\text { of TriModel } \\
(\%)\end{array}$ & $\begin{array}{l}\text { Relative ER } \\
\text { reduction (\%) }\end{array}$ \\
\hline$N R$ & 12.00 & 16.00 & 25.00 \\
\hline$G P C R$ & 14.00 & 20.00 & 30.00 \\
\hline IC & 4.00 & 7.00 & 42.86 \\
\hline$E$ & 3.00 & 5.00 & 40.00 \\
\hline \multicolumn{3}{|c|}{ The average of $\Delta E R$ across all datasets } & 34.47 \\
\hline
\end{tabular}

and TriModel, as the second-best performing method. We also provide the relative error rate reduction based on the two top-performing methods in each dataset. The DTiGEMS + method consistently reduced the relative error rate compared to the other state-of-the-art methods.

Furthermore, we show the practical assessment of the predictive power of DTiGEMS+ for real scenarios of DTI prediction at each drug node. This test is done to show the ability of our model in re-positioning a particular 
drug other than a hub node drug. It should be noted that hub nodes will likely not be the subject of drug research and development as they are likely well-studied. Our procedure goes as follows: first, we calculate the average precision for predicting DTI at each drug, then we average this value over tenfolds. Finally, we calculate mean average precision (MAP) as the mean of tenfolds average precision for each drug across all drug nodes in the graph. We show that DTiGEMS+ archives high MAP values, over NR, GPCR, IC, and E datasets as 0.88, 0.80, 0.91 and 0.88 , respectively. Thus, the overall performance of our model is not likely driven by the hub nodes performance.

\section{DTI prediction and validation of the newly predicted DTIs}

To demonstrate the practical use of our model, we assessed its ability to predict the novel DTIs in each of the benchmark datasets separately. The procedure that we follow to predict novel DTIs is as follows: for each dataset, we first trained our model using all known interactions (positive labels) and split the unknown interactions (negative labels) into training and testing sets for each fold in the tenfold CV. In this manner, we determined if any of the unknown DTI (negative labels) are predicted to be positive DTIs, and then ranked the DTIs predicted to be positive, based on their prediction scores. We only reported and validated the novel DTIs that were not part of the training data (i.e., newly predicted DTIs in the testing data).

To verify the novel DTIs, we manually validated the top 10 ranked newly predicted DTIs for each benchmark dataset. We used biomedical literature and several reference databases, including KEGG [81, 82], DrugBank [83, 84], PubChem [85, 86], CheMBLE [8789], MATADOR [90], SuperTarget [90], Comparative Toxicogenomics Database (CTD) [91, 92], and the annotated database of common toxins and their targets (T3DB) [93]. We found evidence that of the top 10 ranked newly predicted DTIs for each of the 4 benchmark datasets (i.e., for the 40 newly predicted DTIs), 28 DTIs $(70 \%)$ are known interaction. The interaction data was last updated in 2008; this may be the reason why we managed to verify so many of the newly predicted DTIs. Table 5 shows the top novel DTIs for each dataset with the validation evidence for these validated interactions. However, if there is no evidence found in the literature, we marked the evidence as unknown since there is no proof that this interaction exists.

\section{Distinctive characteristics of DTiGEMS+}

Table 4 and Fig. 4 show that DTiGEMS+, TriModel, and DDR are the three top-performing methods, respectively, and all three methods are graph-based. Being graph-based allows these methods to avoid some of the limitations associated with the other methods, and they have a few common characteristics that boost their performance. The main characteristics of these methods are that they formulate the problem as a link prediction in a heterogeneous graph, so they constructed the heterogeneous graph through the integration of multiple information types from different sources. DDR constructed the heterogeneous graph through the integration of multiple similarities from different sources of information, while the TriModel used knowledge graph embedding to infer novel DTIs. DTiGEMS+, on the other hand, kind of fused these methods, by constructing one heterogeneous graph (G1) through the integration of multiple similarities from different sources of information and a second graph (G2) using cosine similarity based on node embeddings generated by applying node2vec on the initial DTI graph $(\boldsymbol{G})$.

Both DTiGEMS+ and DDR integrating multiple similarities should yield a significant improvement in the prediction task. However, some similarities are weak, which means they introduce noise into the data along with the vital information used in the learning and prediction processes. Thus, instead of integrating all similarities, DTiGEMS+ and DDR used similarity selection to identify the optimal subset of similarities that gives optimal results while eliminating the noise. In this regard, DTiGEMS+ used the FSS algorithm (explained in "Similarity-based algorithms" section) to provide useful insights into the optimal subset of similarities for drugs, as well as for target. This algorithm continues to add similarities and only stops when further improvements are no longer visible. Thus, this procedure is time-consuming but provide a higher probability of determining the optimal subset of similarities. On the other hand, to select the optimal similarity subset, DDR calculated entropy values that indicate if the information carried by the similarity matrix is less or more random, then implemented a cut-off to remove similarity matrices carrying weak or random information. The issue here is that even though DDR produced excellent results, the cut-off used could have removed similarity matrices that contain information that contributed to the better performance of DTiGEMS+.

After selecting the optimal subset of similarities, both DTiGEMS + and DDR used an integration function to integrate the similarities. In "Similarity-based algorithms" section, we showed that SNF is the better performing integration function for all datasets, while the AVG function performed the second-best for most datasets except the GPCR dataset, where its performance is identical to SNF. Both DTiGEMS+ and DDR implemented SNF, which not only integrates the similarities but also enforces noise reduction as part of the 
Table 5 Validation of the 10-top ranked newly predicted DTIs for each dataset

\begin{tabular}{|c|c|c|c|c|c|c|}
\hline Data sets & $\#$ & KEGG: Drug ID & Drug name & KEGG: Target ID & Target name & Validation evidence \\
\hline \multirow[t]{10}{*}{ NR } & 1 & D01132 & Tazarotene & hsa6097 & $\begin{array}{l}\text { RORC (RAR Related Orphan } \\
\text { Receptor C) }\end{array}$ & Unknown \\
\hline & 2 & D00182 & Norethindrone & hsa2099 & ESR1 (Estrogen Receptor Alpha) & $\begin{array}{l}\text { PMID: } 27245768 \\
\text { T3DB: T3D4745 }\end{array}$ \\
\hline & 3 & D00075 & Testosterone & hsa5241 & PGR (Progesterone Receptor) & $\begin{array}{l}\text { PMID: } 23229004 \\
\text { PMID: } 23933754 \\
\text { C: CHEMBL386630 }\end{array}$ \\
\hline & 4 & D01132 & Tazarotene & hsa190 & $\begin{array}{l}\text { NROB1 (Nuclear Receptor } \\
\text { Subfamily 0 Group B Member 1) }\end{array}$ & Unknown \\
\hline & 5 & D00094 & Tretinoin & hsa3174 & $\begin{array}{l}\text { HNF4G (Hepatocyte Nuclear } \\
\text { Factor } 4 \text { Gamma) }\end{array}$ & Unknown \\
\hline & 6 & D00554 & Ethinyl estradiol & hsa2100 & ESR2 (Estrogen Receptor 2) & CTD: D004997 \\
\hline & 7 & D00327 & Fluoxymesterone & hsa5241 & PGR (Progesterone Receptor) & Unknown \\
\hline & 8 & D01294 & Ethynodiol diacetate & hsa2100 & ESR2 (Estrogen Receptor 2) & Unknown \\
\hline & 9 & D00299 & Dihydrotachysterol & hsa190 & $\begin{array}{l}\text { NROB1 (Nuclear Receptor } \\
\text { Subfamily O Group B Member } \\
\text { 11) }\end{array}$ & Unknown \\
\hline & 10 & D00094 & Tretinoin & hsa6095 & $\begin{array}{l}\text { RORA (RAR Related Orphan } \\
\text { Receptor A) }\end{array}$ & C: CHEMBL38 \\
\hline \multirow[t]{10}{*}{ GPCR } & 1 & D00283 & Clozapine & hsa1814 & DRD3 (Dopamine Receptor D3) & $\begin{array}{l}\text { C: CHEMBL42 } \\
\text { M: Clozapine (direct) } \\
\text { DB: DB00363 }\end{array}$ \\
\hline & 2 & D02358 & Metoprolol & hsa154 & ADRB2 (Adrenoceptor Alpha 1B) & DB: DB00264 \\
\hline & 3 & D00437 & Nifedipine & hsa152 & $\begin{array}{l}\text { ADRA2C (Adrenergic Receptor } \\
\text { alpha-2C) }\end{array}$ & C: CHEMBL193 \\
\hline & 4 & D00604 & Clonidine hydrochloride & hsa147 & $\begin{array}{l}\text { ADRA1B (Adrenergic Receptor } \\
\text { alpha-1B) }\end{array}$ & DB: DB00575 \\
\hline & 5 & D00255 & Carvedilol & hsa152 & $\begin{array}{l}\text { ADRA2C (Adrenergic Receptor } \\
\text { alpha-2C) }\end{array}$ & DB: DB01136 \\
\hline & 6 & D00451 & Sumatriptan & hsa3363 & $\begin{array}{l}\text { HTR7 (5-Hydroxytryptamine } \\
\text { Receptor 7) }\end{array}$ & Unknown \\
\hline & 7 & D00397 & Tropicamide & hsa1133 & $\begin{array}{l}\text { CHRM5 (Cholinergic Receptor } \\
\text { Muscarinic 5) }\end{array}$ & KG: D00397 \\
\hline & 8 & D00270 & Chlorpromazine & hsa152 & ADRA2C (Adrenoceptor Alpha 2C) & KG: D00270 \\
\hline & 9 & D02250 & Octreotide acetate & hsa6751 & SSTR1 (Somatostatin Receptor 1) & CTD: D015282 \\
\hline & 10 & D01103 & Trospium chloride & hsa1129 & $\begin{array}{l}\text { CHRM2 (Cholinergic Receptor } \\
\text { Muscarinic 2) }\end{array}$ & KG: D01103 \\
\hline \multirow[t]{6}{*}{ IC } & 1 & D00649 & Amiloride hydrochloride & hsa8911 & $\begin{array}{l}\text { CACNA1I (Calcium Voltage-Gated } \\
\text { Channel Subunit Alpha1 I) }\end{array}$ & M: Amiloride (direct) \\
\hline & 2 & D03365 & Nicotine & hsa1137 & $\begin{array}{l}\text { CHRNA4 (Cholinergic Receptor } \\
\text { Nicotinic Alpha } 4 \text { Subunit) }\end{array}$ & $\begin{array}{l}\text { PMID: } 17590520 \\
\text { KG: D03365 } \\
\text { DB: DB00184 }\end{array}$ \\
\hline & 3 & D00775 & Riluzole & hsa2898 & $\begin{array}{l}\text { GRIK2 (Glutamate lonotropic } \\
\text { Receptor Kainate Type Subunit } \\
\text { 2) }\end{array}$ & KG: D00775 \\
\hline & 4 & D00438 & Nimodipine & hsa779 & $\begin{array}{l}\text { CACNA1S (Calcium Voltage-Gated } \\
\text { Channel Subunit Alpha1S) }\end{array}$ & $\begin{array}{l}\text { KG: D00438 } \\
\text { DB: DB00393 }\end{array}$ \\
\hline & 5 & D00726 & Metoclopramide & hsa1138 & $\begin{array}{l}\text { CHRNA5 (Cholinergic Receptor } \\
\text { Nicotinic Alpha } 5 \text { Subunit) }\end{array}$ & Unknown \\
\hline & 6 & D00552 & Benzocaine & hsa6331 & $\begin{array}{l}\text { SCN5A (Sodium Voltage-Gated } \\
\text { Channel Alpha Subunit 5) }\end{array}$ & KG: D00552 \\
\hline
\end{tabular}


Table 5 (continued)

\begin{tabular}{|c|c|c|c|c|c|c|}
\hline Data sets & $\#$ & KEGG: Drug ID & Drug name & KEGG: Target ID & Target name & Validation evidence \\
\hline & 7 & D00542 & Halothane & hsa3736 & $\begin{array}{l}\text { KCNA1(Potassium Voltage-Gated } \\
\text { Channel Subfamily A Member 1) }\end{array}$ & Unknown \\
\hline & 8 & D02098 & Proparacaine hydrochloride & hsa8645 & $\begin{array}{l}\text { KCNK5 (Potassium Two Pore } \\
\text { Domain Channel Subfamily K } \\
\text { Member 5) }\end{array}$ & Unknown \\
\hline & 9 & D01599 & Gliclazide & hsa3758 & $\begin{array}{l}\text { KCNJ1 (Potassium Inwardly } \\
\text { Rectifying Channel Subfamily J } \\
\text { Member 1) }\end{array}$ & Unknown \\
\hline & 10 & D00538 & Zonisamide & hsa6331 & $\begin{array}{l}\text { SCN5A (Sodium Voltage-Gated } \\
\text { Channel Alpha Subunit 5) }\end{array}$ & $\begin{array}{l}\text { DB: DB00909 } \\
\text { KG: D00538 }\end{array}$ \\
\hline \multirow[t]{10}{*}{ E } & 1 & D00437 & Nifedipine & hsa1559 & $\begin{array}{l}\text { CYP2C9 (Cytochrome P450 Family } \\
2 \text { Subfamily C Member 9) }\end{array}$ & $\begin{array}{l}\text { CTD: D009543 } \\
\text { PMID: } 9929518\end{array}$ \\
\hline & 2 & D00574 & Aminoglutethimide & hsa1589 & $\begin{array}{l}\text { CYP21A2 (Cytochrome P450 } \\
\text { Family } 21 \text { Subfamily A Member } \\
\text { 2) }\end{array}$ & $\begin{array}{l}\text { M: Aminoglutethimide } \\
\text { (indirect) } \\
\text { PMID: } 8201961\end{array}$ \\
\hline & 3 & D00410 & Metyrapone & hsa1583 & $\begin{array}{l}\text { CYP11A1 (Cytochrome P450 } \\
\text { Family } 11 \text { Subfamily A Member } \\
\text { 1) }\end{array}$ & CTD: D008797 \\
\hline & 4 & D00437 & Nifedipine & hsa1585 & $\begin{array}{l}\text { CYP11B2 (Cytochrome P450 } \\
\text { Family } 11 \text { Subfamily B Member } \\
\text { 2) }\end{array}$ & $\begin{array}{l}\text { M: Nifedipine- (indirect) } \\
\text { CTD: D009543 }\end{array}$ \\
\hline & 5 & D00410 & Metyrapone & hsa1543 & $\begin{array}{l}\text { CYP1A1 (Cytochrome P450 Family } \\
1 \text { Subfamily A Member) }\end{array}$ & PMID: 9512490 \\
\hline & 6 & D03670 & Deferoxamine & hsa51302 & $\begin{array}{l}\text { CYP39A1 (Cytochrome P450 } \\
\text { Family } 39 \text { Subfamily A Member } \\
\text { 1) }\end{array}$ & Unknown \\
\hline & 7 & D00043 & Isoflurophate & hsa1991 & $\begin{array}{l}\text { ELANE (Elastase, Neutrophil } \\
\text { Expressed) }\end{array}$ & $\begin{array}{l}\text { M: Diisopropylfluorophos- } \\
\text { phate (indirect) }\end{array}$ \\
\hline & 8 & D00947 & Linezolid & hsa4129 & MAOB (Monoamine Oxidase B) & CTD: D000069349 \\
\hline & 9 & D03670 & Deferoxamine & hsa4353 & MPO (Myeloperoxidase) & $\begin{array}{l}\text { M: Desferrioxamine } \\
\text { (indirect) }\end{array}$ \\
\hline & 10 & D05458 & Phentermine & hsa4128 & MAOA (Monoamine Oxidase A) & $\begin{array}{l}\text { KG: D05458 } \\
\text { DB: DB00191 }\end{array}$ \\
\hline
\end{tabular}

C: ChEMBL; CTD: comparative toxicogenomics database; DB: DrugBank; M: MATADOR; KG: KEGG; PMID: PubMed;T3DB: toxin and toxin-target database

integration process. That is, the low-weight edges that represent weak similarity have disappeared, captures the most informative features. Thus, the better performance seen with both DTiGEMS + and DDR compared to other methods, may also be contributed to by the implementation of SNF, which is the only integration function that enforces noise reduction. Additional file 1: Table S2 provides the set of drug-drug similarities as well as the set of target-target similarities that are selected and then fused, as well as the best-performing integration function/s.

For DTiGEMS+, the KNN that performs noise reduction is not only a component of SNF, we also used KNN (on the drug-drug similarity, target-target similarity) augmented with DTI to construct the graph fed to node2 vec. In this manner, the graph used for generating the embeddings needed to construct graph $\boldsymbol{G} 2$ only provides the informative edges for the generation of good quality graph embeddings that capture meaningful proximity information between nodes. Another advantage of applying node2vec on the graph that kept just the KNN similar drugs and targets, is that it reduced the node2vec model running time since the number of edges for each drug similarity graph (and target similarity graph) reduced from $n(n-1) / 2$ to $\left(K^{*} n\right)$ where $\mathrm{n}$ is the number of drugs. Second, we computed two cosine similarity matrices based on node2vec feature representations for each drug pair and target pair because it gives unique similarity between nodes that carry meaningful topological, relational, and structural information. So, even if the two similar nodes are not close based on the Euclidean distance, their feature vectors could still have a small angle between them, indicating their high similarity. 
Formulating a new graph with these new similarities provided a better representation of the graph that was used to extract the path score features. These factors may provide DTiGEMS + with an advantage over the TriModel, as they may be contributing to the capturing of quality embeddings due to noise reduction and or our method identifying potential DTIs excluded from TriModel. It is important to mention that we did the experiments by feeding the whole graph without removing any edge to node2vec and the results of AUPR were close to or lower than the experiment results when we used KNN drugs similarity and KNN targets similarity which means removing weak edges is not causing that we are missing important information.

DTiGEMS+ has another advantage over other graphbased methods that used path structure scores as their model features, such as in [21,34]. We analyzed these features and recognized that the $\mathrm{D}-\mathrm{T}-\mathrm{D}-\mathrm{T}$ path structure, for example, is not based on informative features. That is, the D-T-D-T path structure is generated only using the information of known DTIs, which is limited in number, causing these features to be sparse. So, we removed the sum and max features for such path structure for both graph $\mathbf{G 1}$ and $\mathbf{G 2}$.

At the classification stage, some other methods directly apply RF as it is a recognized prediction tool that runs efficiently on large datasets, and is less prone to overfitting. However, for DTiGEMS+, we accessed the performance of three different classifiers (RF, NN, Adaboost) on each dataset, then chose the best performing classifier for each dataset. NN classifier performed the best for the NR dataset. We expected this result as the NN classifier is known to perform better when modeling high volatility data, which is the case for the NR dataset due to its small size. Nonetheless, ensemble learning techniques such as RF and Adaboost have proven efficacy when dealing with DTI prediction problems [8, 21, 34, 94, 95]. The RF classifier combines several individual classifiers that vote and nominates the majority voting class as the prediction class. On the other hand, the Adaboost classifier creates a robust classifier from several weak classifiers by building a first model from the training data, and then create a second model that tries to correct the errors in the first model; this process is repeated until the prediction performance of the training data is improved. One advantage of RF over Adaboost is that RF runs in parallel while Adaboost runs sequentially, so RF is a much faster classification process. Nonetheless, Adaboost performance was very close to $\mathrm{NN}$ for the NR dataset (less by $1 \%$ in AUPR). Moreover, Adaboost performed better than both RF and NN for the other datasets (GPCR, IC, and E). It is worth noting that the RF classifier was, however, competitive for IC and E datasets (very close AUPR) with a more significant number of known interactions.

\section{Conclusion}

Our work introduced a novel computational method for drug-target interactions prediction named (DTiGEMS+). DTiGEMS + integrated different techniques from ML, graph embedding, graph mining, and similarity-based methods. That is, (1) graph embedding was used in node2vec feature representation to benefit from the network topology and structural features, (2) graph mining was used to extract path score features, (3) similarity-based techniques were used to select and integrate multiple similarities from different information sources, and finally, (4) ML for classification. The novelty of our method lies in generating graph-based path score features from two graphs that were constructed using the same DTIs but using different types of similarity matrices that carry unique information. For example, Graph $\mathbf{G 1}$ used to fuse the drug-drug and target-target similarities carry complementary information from chemical structure and side effects for drugs, etc., and gene ontology and aminoacid sequences for target proteins, etc., while graph, G2 used drug-drug and target-target cosine similarities of generated embedding that carry topological information. DTiGEMS + proved its efficiency by outperforming seven state-of-the-art methods using several evaluation metrics, and by predicting novel DTIs that were validated using published literature and different online databases.

For further improvements to DTiGEMS+, we suggest applying different embeddings techniques, integrating more similarity measures from more sources, and generating more graph-based features. Also, as the current implementation of DTiGEMS+ constructs negative DTIs from the random pairing of drugs and targets that have no edges (unknown interaction), in the future, we plan to extend the functionality of our method to create a reliable set of negative DTIs following [96]. Furthermore, we intend to use our method to predict DTIs for new drugs or new targets. Some potential extensions of our work include applying DTiGEMS + to different graphs (i.e., network) formulated as a link prediction problem. Popular examples of link prediction in the bioinformatics field include but are not limited to, drug-drug interactions prediction, drug-disease interactions prediction, genedisease association prediction. Another extension would be amending DTiGEMS + to address DTIs as a regression problem for the prediction of the binding affinity between drugs and their target proteins. 


\section{Supplementary information}

Supplementary information accompanies this paper at https://doi. org/10.1186/s13321-020-00447-2.

Additional file 1. Additional Tables.

\section{Abbreviations}

DTIs: Drug-target interactions; 3D: 3-dimensional; ML: Machine learning; DL: Deep learning; CNN: Convolutional neural network; E: Enzyme; IC: Ion channel; GPCR: G-protein-coupled receptor; NR: Nuclear receptor; GIP: Gaussian interaction profile; SW: Smith-Waterman; GO: Gene ontology; PPI: Protein-protein interaction; SNF: Similarity network fusion; KNN: K-nearest neighbor; FSS: Forward similarity selection; DD sim: Drug-drug similarity matrix; TT sim: Targettarget similarity matrix; SMOTE: Synthetic Minority Over-sampling Technique; NN: Neural network; MLP: Multilayer perceptron; Adaboost: Adaptive boosting; RF: Random forest; ROC: Receiver operating characteristic; AUC: The area under ROC curve; AUPR: The area under the precision-recall curve; FPR: False positive rate; TPR: True positive rate; ER: Error rate; CV: Cross validation; MAP: Mean average precision; P-value: Probability value.

\section{Acknowledgements}

The research reported in this publication was supported by the King Abdullah University of Science and Technology (KAUST).

\section{Authors' contributions}

The study is conceptualized and designed by VBB, ME and MAT. MAT implemented the code, and wrote the manuscript. MT and SA designed the figures. MAT, VBB, ME, RSO, HA and SA contributed to the discussions. RSO and $H A$ validated and analyzed the novel interactions and contributes in results and discussion section. $M E, X G, T G$, and $V B B$ revised/edited the manuscript. All authors read and approved the final manuscript.

\section{Funding}

The research reported in this publication was supported by King Abdullah University of Science and Technology (KAUST) through the Awards Nos. BAS/1/1606-01-01, BAS/1/1059-01-01, BAS/1/1624-01-01, FCC/1/1976-17-01, and FCC/1/1976-26-01.

\section{Availability of data and materials}

The source code and datasets used in the paper can be found in the: https:// github.com/MahaThafar/Drug-Target-Interaction-Prediciton-Method.

\section{Competing interests}

The authors have declared that no conflict of interests exist.

\begin{abstract}
Author details
${ }^{1}$ Computer, Electrical and Mathematical Sciences and Engineering Division (CEMSE), Computational Bioscience Research Center (CBRC), King Abdullah University of Science and Technology (KAUST), Thuwal, Kingdom of Saudi Arabia. ${ }^{2}$ Collage of Computers and Information Technology, Taif University, Taif, Kingdom of Saudi Arabia. ${ }^{3}$ The Jackson Laboratory for Genomic Medicine, Farmington, CT, USA. ${ }^{4}$ Faculty of Computing and Information Technology, King Abdulaziz University, Jeddah, Kingdom of Saudi Arabia. ${ }^{5}$ Biological and Environmental Sciences and Engineering Division (BESE), King Abdullah University of Science and Technology (KAUST), Thuwal, Kingdom of Saudi Arabia.
\end{abstract}

Received: 10 December 2019 Accepted: 16 June 2020

Published online: 29 June 2020

\section{References}

1. DiMasi JA, Hansen RW, Grabowski HG (2003) The price of innovation: new estimates of drug development costs. J Health Econ 22(2):151-185

2. Yıldırım MA et al (2007) Drug-target network. Nat Biotechnol 25:1119

3. Ashburn TT, Thor KB (2004) Drug repositioning: identifying and developing new uses for existing drugs. Nat Rev Drug Discov. 3(8):673-683
4. Cheng AC et al (2007) Structure-based maximal affinity model predicts small-molecule druggability. Nat Biotechnol 25(1):71-75

5. Alonso H, Bliznyuk AA, Gready JE (2006) Combining docking and molecular dynamic simulations in drug design. Med Res Rev 26(5):531-568

6. Wang Ket al (2013) Prediction of drug-target interactions for drug repositioning only based on genomic expression similarity. PLoS Comput Biol 9(11):e1003315

7. Tropsha A (2010) Best practices for QSAR model development, validation, and exploitation. Mol Inform. 29(6-7):476-488

8. Rayhan F et al (2017) iDTI-ESBoost: identification of drug target interaction using evolutionary and structural features with boosting. Sci Rep. 7(1):17731

9. Pathak S, Cai X. Ensemble learning algorithm for drug-target interaction prediction. 2017 IEEE 7th international conference on computational advances in Bio and medical sciences (ICCABS), 2017

10. Nascimento ACA, Prudêncio RBC, Costa IG (2016) A multiple kernel learning algorithm for drug-target interaction prediction. BMC Bioinform $17: 46$

11. He T et al (2017) SimBoost: a read-across approach for predicting drugtarget binding affinities using gradient boosting machines. J Cheminform $9(1): 1-4$

12. Naveed $\mathrm{H}$ et al (2015) An integrated structure-and system-based framework to identify new targets of metabolites and known drugs. Bioinformatics 31(24):3922-3929

13. Zong $\mathrm{N}$ et al (2017) s. Bioinformatics 33(15):2337-2344

14. Wang $L$ et al (2017) Computational methods for the prediction of drugtarget interactions from drug fingerprints and protein sequences by stacked auto-encoder deep neural network. Bioinform Res Appl. Springer, Cham, pp 46-58

15. Gao, K.Y., et al., Interpretable Drug Target Prediction Using Deep Neural Representation, In: IJCAI. 2018, 3371-3377

16. Tsubaki M, Tomii K, Sese J (2019) Compound-protein interaction prediction with end-to-end learning of neural networks for graphs and sequences. Bioinformatics 35(2):309-318

17. Cheng F et al (2012) Prediction of drug-target interactions and drug repositioning via network-based inference. PLoS Comput Biol 8(5):e1002503

18. Emig D et al (2013) Drug target prediction and repositioning using an integrated network-based approach. PLoS ONE 8(4):e60618

19. Ba-Alawi W et al (2016) DASPfind: new efficient method to predict drug-target interactions. J Cheminform. 8:15

20. Luo Y et al (2017) A network integration approach for drug-target interaction prediction and computational drug repositioning from heterogeneous information. Nat Commun. 8(1):573

21. Olayan RS, Ashoor H, Bajic VB (2018) DDR: efficient computational method to predict drug-target interactions using graph mining and machine learning approaches. Bioinformatics 34(7):1164-1173

22. Anusuya $S$ et al (2018) Drug-target interactions: prediction methods and applications. Curr Protein Pept Sci 19(6):537-561

23. Ding $\mathrm{H}$ et al (2014) Similarity-based machine learning methods for predicting drug-target interactions: a brief review. Brief Bioinform 15(5):734-747

24. Nath A, Kumari P, Chaube R (2018) Prediction of human drug targets and their interactions using machine learning methods: current and future perspectives. Methods Mol Biol 1762:21-30

25. Ezzat A et al (2018) Computational prediction of drug-target interactions using chemogenomic approaches: an empirical survey. Brief Bioinform 20(4):1337-1357

26. Sachdev K, Gupta MK (2019) A comprehensive review of feature based methods for drug target interaction prediction. J Biomed Inform 93:103159

27. Zhou L et al (2019) Revealing drug-target interactions with computational models and algorithms. Molecules 24(9):1714

28. Zhang W et al (2019) Recent advances in the machine learning-based drug-target interaction prediction. Curr Drug Metab 20(3):194-202

29. Thafar M, Raies AB, Albaradei S, Essack M, Bajic VB (2019) Comparison study of computational prediction tools for drug-target binding affinities. Front Chem 7:782

30. Kurgan L, Wang C (2018) Survey of similarity-based prediction of drugprotein interactions. Curr Med Chem. https://doi.org/10.2174/09298 67326666190808154841 
31. Yamanishi Y et al (2010) Drug-target interaction prediction from chemical, genomic and pharmacological data in an integrated framework. Bioinformatics 26(12):i246-i254

32. Bleakley K, Yamanishi Y (2009) Supervised prediction of drug-target interactions using bipartite local models. Bioinformatics 25(18):2397-2403

33. Yamanishi $Y$ et al (2008) Prediction of drug-target interaction networks from the integration of chemical and genomic spaces. Bioinformatics 24(13):i232-i240

34. Xuan P et al (2019) Gradient boosting decision tree-based method for predicting interactions between target genes and drugs. Front Genet. $10: 459$

35. Tabei $Y$ et al (2019) Network-based characterization of drug-protein interaction signatures with a space-efficient approach. BMC Syst Biol 13(Suppl 2):39

36. Aghakhani S, Qabaja A, Alhajj R (2018) Integration of k-means clustering algorithm with network analysis for drug-target interactions network prediction. Int J Data Mining Bioinform 20(3):185

37. Bansal A, Srivastava PA, Singh TR (2018) An integrative approach to develop computational pipeline for drug-target interaction network analysis. Sci Rep. 8(1):10238

38. Seal A, Ahn YY, Wild DJ (2015) Optimizing drug-target interaction prediction based on random walk on heterogeneous networks. J Cheminform. 7(1):40

39. Liu $Y$ et al (2016) Neighborhood regularized logistic matrix factorization for drug-target interaction prediction. PLoS Comput Biol 12(2):e1004760

40. Yan X-Y, Li R-Z, Kang L (2019) Prediction of drug-target interaction with graph regularized non-negative matrix factorization. J Phys Conf Ser 1237:032017

41. Cui Z et al (2019) L-GRMF: an improved graph regularized matrix factorization method to predict drug-target interactions. BMC Bioinform 20(Suppl 8):287

42. Xia L-Y et al (2019) Improved prediction of drug-target interactions using self-paced learning with collaborative matrix factorization. J Chem Inf Model 59(7):3340-3351

43. Hao M, Bryant SH, Wang Y (2017) Predicting drug-target interactions by dual-network integrated logistic matrix factorization. Sci Rep. 7:40376

44. Goyal P, Ferrara E (2018) Graph embedding techniques, applications, and performance: a survey. Knowl Based Syst 151:78-94

45. Cai H, Zheng WW, Chang KC (2018) A comprehensive survey of graph embedding: problems, techniques, and applications. IEEE Trans Knowl Data Eng 30(9):1616-1637

46. Mohamed SK, Nováček V, Nounu A (2019) Discovering protein drug targets using knowledge graph embeddings. Bioinformatics 36(2):603-610

47. Alshahrani M et al (2017) Neuro-symbolic representation learning on biological knowledge graphs. Bioinformatics 33(17):2723-2730

48. Tian K et al (2016) Boosting compound-protein interaction prediction by deep learning. Methods 110:64-72

49. You J, McLeod RD, Hu P (2019) Predicting drug-target interaction network using deep learning model. Comput Biol Chem 80:90-101

50. Wan F et al (2019) NeoDTI: neural integration of neighbor information from a heterogeneous network for discovering new drug-target interactions. Bioinformatics 35(1):104-111

51. Lee I, Keum J, Nam H (2019) DeepConv-DTI: prediction of drug-target interactions via deep learning with convolution on protein sequences. PLoS Comput Biol 15(6):e1007129

52. Kulin M et al (2018) End-to-end learning from spectrum data: a deep learning approach for wireless signal identification in spectrum monitoring Appl. IEEE Access 6:18484-18501

53. Öztürk H, Özgür A, Ozkirimli E (2018) DeepDTA: deep drug-target binding affinity prediction. Bioinformatics 34(17):i821-i829

54. Rogers D, Hahn M (2010) Extended-connectivity fingerprints. J Chem Inf Model 50(5):742-754

55. Klambauer G et al (2015) Rchemcpp: a web service for structural analoging in ChEMBL. Drugbank and the connectivity map. Bioinformatics 31(20):3392-3394

56. Kashima H, Tsuda K, Inokuchi A, Marginalized kernels between labeled graphs. In: Proceedings of the 20th international conference on machine learning, 2003
57. Ralaivola L et al (2005) Graph kernels for chemical informatics. Neural Netw. 18(8):1093-1110

58. Michael Mahr, GK, Rchemcpp. 2017, Bioconductor

59. Tenenbaum D (2019) KEGGREST: client-side REST access to KEGG. R Package Version 1.24.0. Fred Hutchinson Cancer Research Center, Seattle, WA, USA

60. Cao $Y$ et al (2008) ChemmineR: a compound mining framework for $R$ Bioinformatics 24(15):1733-1734

61. Kuhn M et al (2010) A side effect resource to capture phenotypic effects of drugs. Mol Syst Biol. 6:343

62. Takarabe M et al (2012) Drug target prediction using adverse event report systems: a pharmacogenomic approach. Bioinformatics 28(18):i611-i618

63. van Laarhoven T, Nabuurs SB, Marchiori E (2011) Gaussian interaction profile kernels for predicting drug-target interaction. Bioinformatics 27(21):3036-3043

64. Smith SB et al (2012) Identification of common biological pathways and drug targets across multiple respiratory viruses based on human host gene expression analysis. PLoS ONE 7(3):e33174

65. Palme J, Hochreiter S, Bodenhofer U (2015) KeBABS: an R package for kernel-based analysis of biological sequences: fig. 1. Bioinformatics 31(15):2574-2576

66. Ovaska K, Laakso M, Hautaniemi S (2008) Fast gene ontology based clustering for microarray experiments. BioData Min. 1(1):11

67. Wang B et al (2014) Similarity network fusion for aggregating data types on a genomic scale. Nat Methods 11(3):333-337

68. Grover A, Leskovec J (2016) node2vec: scalable Feature Learning for Networks. KDD 2016:855-864

69. Fu G et al (2016) Predicting drug target interactions using meta-pathbased semantic network analysis. BMC Bioinformatics 17:160

70. Liu A, Ghosh J, Martin CE (2007) Generative oversampling for mining imbalanced datasets. In: Proceedings of the 2007 international conference on data mining (DMIN), pp 66-72

71. Kovács G (2019) Smote-variants: a python implementation of 85 minority oversampling techniques. Neurocomputing 366:352-354

72. Lemaître G, Nogueira F, Aridas CK (2017) Imbalanced-learn: a python toolbox to tackle the curse of imbalanced datasets in machine learning. J Mach Learn Res. 18(1):559-563

73. Agatonovic-Kustrin S, Beresford R (2000) Basic concepts of artificial neural network (ANN) modeling and its application in pharmaceutical research. J Pharm Biomed Anal 22(5):717-727

74. Kam HT (1995) Random decision forest. In: Proceedings of the 3rd international conference on document analysis and recognition, vol 1416, pp 278-282

75. Freund Y, Schapire RE (1997) A decision-theoretic generalization of on-line learning and an application to boosting. J Comput Syst Sci 55(1):119-139

76. Pedregosa F et al (2011) Scikit-learn: machine Learning in Python. J Mach Learn. Res. 12:2825-2830

77. Davis J, Goadrich M. The relationship between precision-recall and ROC Curves. In: Proceedings of the 23rd international conference on machine learning. 2006, 233-240

78. Powers DM, Evaluation: from precision, recall and F-measure to ROC, informedness, markedness and correlation. 2011

79. Ezzat A, Zhao P, Wu M, Li XL, Kwoh CK (2016) Drug-target interaction prediction with graph regularized matrix factorization. IEEE/ACM Trans Comput Biol Bioinform 14(3):646-656

80. Mei J-P et al (2013) Drug-target interaction prediction by learning from local information and neighbors. Bioinformatics 29(2):238-245

81. Kanehisa M et al (2006) From genomics to chemical genomics: new developments in KEGG. Nucleic Acids Res 34(Database issue):D354-D357

82. Kanehisa M et al (2017) KEGG: new perspectives on genomes, pathways, diseases and drugs. Nucleic Acids Res 45(D1):D353-D361

83. Wishart DS et al (2008) DrugBank: a knowledgebase for drugs, drug actions and drug targets. Nucleic Acids Res 36(Database issue):D901-D906

84. Wishart DS et al (2018) DrugBank 5.0: a major update to the DrugBank database for 2018. Nucleic Acids Res 46:D1074-D1082

85. Bolton EE et al (2008) Chapter 12-PubChem: integrated platform of small molecules and biological activities. In: Wheeler RA, Spellmeyer DC (eds) Annual reports in computational chemistry. Elsevier, Amsterdam, pp 217-241 
86. Kim S et al (2016) PubChem substance and compound databases. Nucleic Acids Res 44(D1):D1202-D1213

87. Bento AP et al (2014) The ChEMBL bioactivity database: an update. Nucleic Acids Res 42(Database issue):D1083-D1090

88. Gaulton A et al (2012) ChEMBL: a large-scale bioactivity database for drug discovery. Nucleic Acids Res 40(Database issue):D1100-D1107

89. Gaulton A et al (2017) The ChEMBL database in 2017. Nucleic Acids Res 45(D1):D945-D954

90. Günther S et al (2008) SuperTarget and Matador: resources for exploring drug-target relationships. Nucleic Acids Res 36(Database issue):D919-D922

91. Davis AP et al (2017) The cComparative toxicogenomics database: update 2017. Nucleic Acids Res 45(D1):D972-D978

92. Davis AP et al (2009) Comparative toxicogenomics database: a knowledgebase and discovery tool for chemical-gene-disease networks. Nucleic Acids Res 37(suppl_1):D786-D792
93. Lim E et al (2010) T3DB: a comprehensively annotated database of common toxins and their targets. Nucleic Acids Res 38(Database issue):D781-D786

94. Coelho ED, Arrais JP, Oliveira JL (2016) Computational discovery of putative leads for drug repositioning through drug-target interaction prediction. PLoS Comput Biol 12(11):e1005219

95. Yu H et al (2012) A systematic prediction of multiple drug-target interactions from chemical, genomic, and pharmacological data. PLoS ONE 7(5):e37608

96. Mervin LH et al (2015) Target prediction utilising negative bioactivity data covering large chemical space. J Cheminform 7:51

\section{Publisher's Note}

Springer Nature remains neutral with regard to jurisdictional claims in published maps and institutional affiliations. 